\title{
Évolution des séquences "signal de recombinaison " dans le locus de la région variable de la chaîne lourde des immunoglobulines
}

\author{
Alexandre Hassanin
}

Les locus des immunoglobulines (Ig) et des récepteurs des cellules $T(T c R)$ sont propres aux vertébrés et présentent une dynamique évolutive exceptionnelle dont les modalités restent cependant à découvrir. Les gènes des Ig et TcR sont assemblés au cours du développement des lymphocytes, à partir des segments codants $V$, D, et $J$, par un mécanisme spécifique appelé recombinaison $V(D) J$. Ce mécanisme très particulier fait intervenir de courtes séquences cibles adjacentes aux segments codants appelées séquences «signal de recombinaison» (SSR). Des travaux récents ont montré que la recombinaison $V(D) J$ pouvait avoir lieu dans les cellules germinales des poissons cartilagineux [12]. Si un tel mécanisme était avéré chez les mammifères, il pourrait expliquer les importantes restructurations observées dans les locus des Ig et des TcR au cours de l'évolution. Dans le cadre de cette hypothèse, on s'attendrait alors à ce que les segments codants et les SSR n'aient pas toujours évolué comme une unité fonctionnelle. Autrement dit, des analyses phylogénétiques menées indépendamment sur

\begin{abstract}
les segments codants et leurs SSR adjacentes devraient montrer des histoires évolutives différentes.

L'étude du locus de la région variable de la chaîne lourde des Ig a permis de définir quatre types de SSR chez l'homme : H1, H2, H3 et H5. L'analyse des séquences disponibles dans les banques de données a indiqué que les autres mammifêres ne présentaient pas de types de SSR autres que ceux découverts chez l'homme. Les analyses phylogénétiques menées sur les segments codants VH et leurs SSR adjacentes ont montré qu'il y a eu remplacement d'une SSR de type H2 par une SSR de type H3 au cours de l'évolution des mammifêres. Deux modèles sont proposés pour rendre compte de ce remplacement : le premier implique un crossing-over inégal, le second fait intervenir une activation de la recombinaison $V(D) J$ dans les cellules germinales. Ce remplacement semble avoir été sélectionné positivement au cours de l'évolution des mammifères car les SSR H3 apparaissent plus efficaces pour la recombinaison $V(D) J$ que les SSR H2.
\end{abstract}

e système immunitaire des vertébrés produit un vaste répertoire d'immunoglobulines (Ig) et de récepteurs des cellules $\mathrm{T}$ ( TcR: T cell receptor) capables de reconnaître un très large spectre d'antigènes. Une molécule d'Ig est un tétramère polypeptidique composé de deux chaînes légères (L) identiques et de deux chaînes lourdes (H) identiques (figure 1). Une molécule de TcR est un hétérodimère composé soit d'une chaîne $\alpha$ et d'une chaîne $\beta$, soit d'une chaîne $\gamma$ et d'une chaîne $\delta$. Les chaînes polypeptidiques des Ig et des TcR sont constituées d'une région variable responsable des interactions avec l'antigène et d'une région constante. La région constante est codée par un seul segment de gène. La région variable est codée par une séquence d'ADN assemblée soit à partir de deux segments de gène (un segment V [variable] et un segment J [jonction]) dans le cas des chaînes L, $\alpha$ et $\gamma$, soit à partir de trois segments de gène ( $\mathrm{V}, \mathrm{D}$ [diversité] et $\mathrm{J}$ ) dans le cas des chaînes $H, \beta$ et $\delta$. Dans le génome des vertébrés, il existe un grand nombre de segments V, D et J et, en théorie, chaque segment $\mathrm{V}$ peut être combiné avec n'importe quel segment D et n'importe quel segment J. Ce mécanisme combinatoire, appelé recombinaison VDJ, 
permet d'obtenir une très grande diversité de réarrangements. Ces réarrangements s'effectuent uniquement dans les lignées lymphocytaires $\mathrm{B}$ et $\mathrm{T}$ et permettent l'établissement des répertoires somatiques des Ig et TcR, propres à chaque individu [1, 2].

Les réarrangements entre les éléments $\mathrm{V}, \mathrm{D}$ et J sont réalisés grâce à de courtes séquences cibles, appelées séquences "signal de recombinaison » (SSR), situées immédiatement en 3' des segments $V$, en 5' et 3' des segments D, et en 5' des segments J (figure 1). Les SSR sont composées de deux motifs très conservés (un hepta-

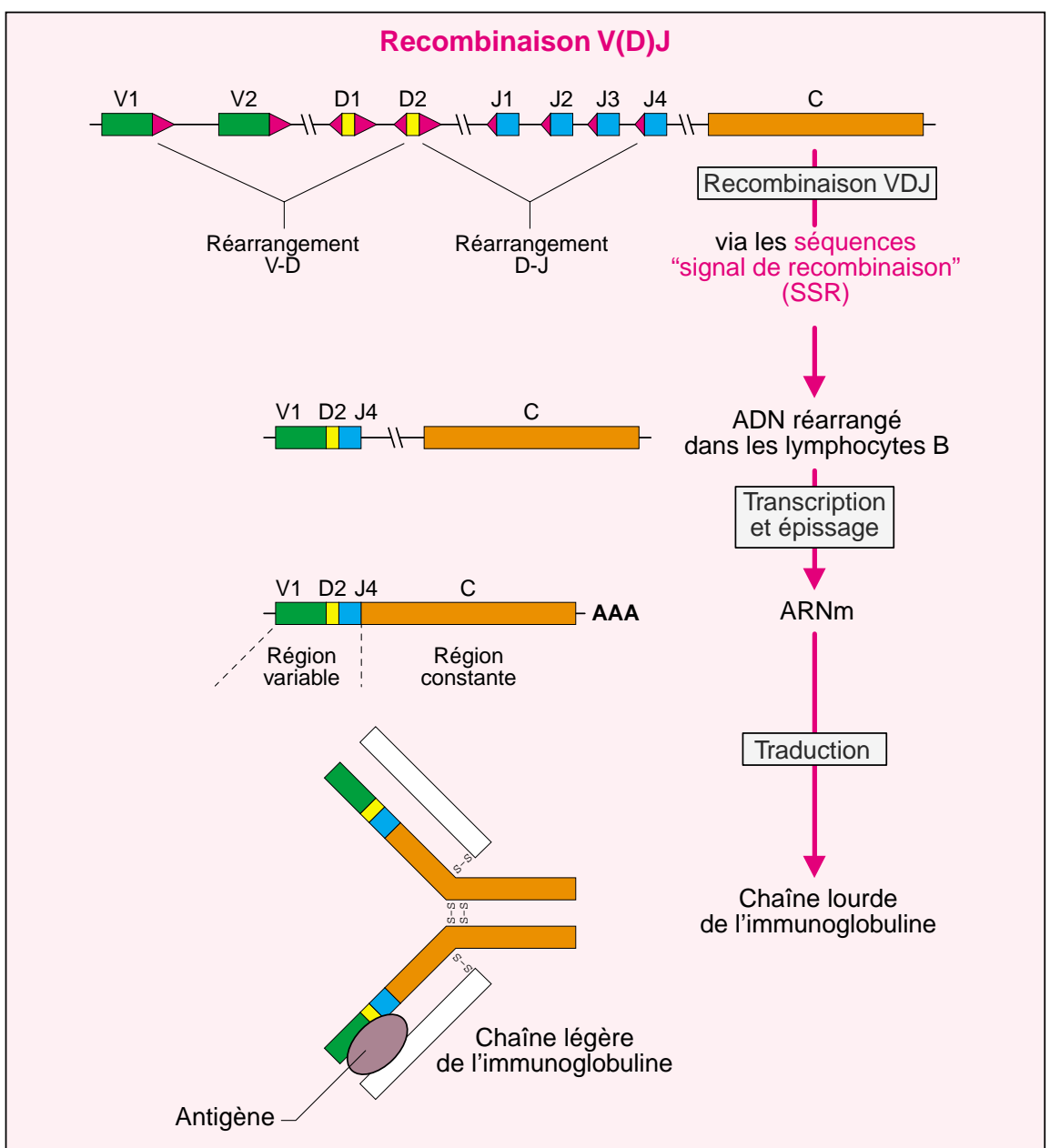

Figure 1. La recombinaison VDJ dans le locus de la chaîne lourde des immunoglobulines. Les séquences "signal de recombinaison" (SSR) sont représentées sous la forme d'un triangle rouge; celles situées en $3^{\prime}$ des segments $V$ et $D$ sont caractérisées par un espaceur de 23 pb alors que celles localisées en $5^{\prime}$ des segments $D$ et $J$ présentent un espaceur de $12 \mathrm{pb}$. Généralement, la recombinaison a lieu entre des SSR de tailles différentes et orientées en sens inverse (règle 12/23) [1, 2].

puisque le locus de la région variable a été complètement séquencé [3]. Une étude restreinte aux 39 segments $\mathrm{VH}$ fonctionnels du locus humain a permis de définir quatre types de SSR, appelés H1, H2, H3, et $\mathrm{H} 5$ [4]. Ces quatre types de SSR sont facilement différentiables en comparant soit les espaceurs de $23 \mathrm{pb}$ des $\mathrm{SSR}$, soit les régions non fonctionnelles situées en 3' des SSR (figure 2). Il est possible d'aligner les régions 3'adjacentes des SSR lorsque les séquences comparées appartiennent à un même type de SSR. En revanche, il s'avère impossible de les aligner lorsque les séquences comparées appartiennent à des types de SSR différents.

Toutes les SSR associées aux segments VH de mammifères appartiennent aux types $\mathrm{H} 1$ et $\mathrm{H} 3$ mis en évidence dans le locus VH humain. Le type $\mathrm{H} 1$ a été retrouvé uniquement chez la souris, alors que le type H3 est beaucoup plus répandu: il a été détecté chez des cétartiodactyles (bœuf, mouton, chameau et porc), un lagomorphe (lapin), des primates (chimpanzé, gorille et macaque) et un rongeur (souris). Aucune SSR de mammifère ne ressemble aux SSR découvertes chez les oiseaux, les reptiles, les amphibiens et les poissons. Ce résultat s'explique probablement par la longue période de temps qui s'est écoulée depuis la séparation des principaux groupes de vertébrés, ce qui a entraîné l'accumulation d'un grand nombre de mutations dans les régions utilisées pour définir les différents types de SSR (l'espaceur et la région 3'-adjacente de la SSR), obscurcissant ainsi le signal phylogénétique ancien.

\section{Le modèle d'évolution birth-and-death}

Le modèle d'évolution birth-and-death postule que les nouveaux segments VH sont créés par duplication; certains sont maintenus sous forme fonctionnelle dans le génome, d'autres sont éliminés ou deviennent des pseudogènes à la suite de mutations délétères $[5,6]$. Pour donner naissance à un nouveau segment de gène fonctionnel, le fragment d'ADN dupliqué doit inclure la séquence $\mathrm{VH}$ 


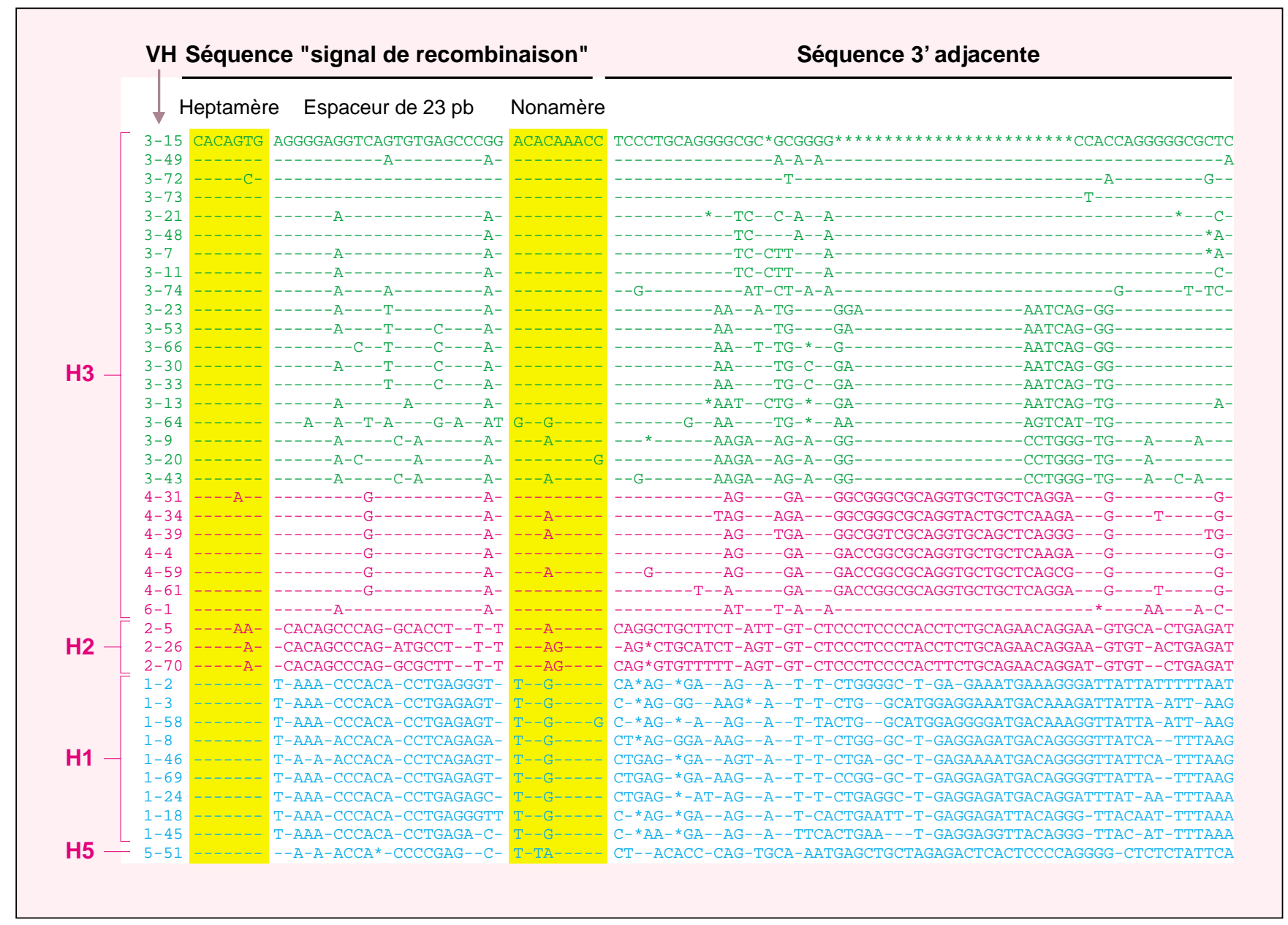

Figure 2. Alignement des 39 SSR fonctionnelles du locus VH humain. L'alignement inclut les trois régions des SSR (heptamère, espaceur de 23 nucléotides et nonamère) ainsi que la séquence 3'-adjacente. Toutes ces données sont tirées de la séquence complète du locus VH humain [3]. Chez I'Homme, les segments de gène VH ont été classés en sept familles (la famille 7 n'est pas représentée ici car aucun segment fonctionnel n'y a été décrit) qui ont été rassemblées dans trois groupes majeurs ( $A$ en bleu, $B$ en rouge et $C$ en vert) [5-7]. Le premier chiffre de chaque nom de segment VH fait référence à sa famille d'appartenance, le second chiffre indique sa position sur le chromosome 14 [3]. Pour la nomenclature des SSR, la lettre $H$ fait référence à I'Homme alors que le chiffre indique la famille VH la plus représentative de cet ensemble [4]. Le type H3 est le plus commun avec 26 membres correspondant aux familles $V_{H} 3,4$ et 6. Le type $H 2$ est associé à la famille $V_{H} 2$. Le type $H 1$ inclut tous les représentants de la famille $V H$ 1. Le type $H 5$ ne contient que $V_{H}$ 5-51.

Figure 3. Arbre phylogénétique des segments de gène $\mathrm{VH}$ de mammifères. Les analyses phylogénétiques ont été réalisées avec le logiciel PAUP 3.1.1 [15] en examinant l'exon 2 (261 pb) de 85 segments VH de mammifères. L'arbre correspond au consensus strict des 54 arbres les plus courts (longueur = 396410 pas) obtenus en parcimonie pondérée $[16,17]$. La robustesse des regroupements a été estimée par la méthode du Bootstrap [18]: les valeurs obtenues après 100 réplications sont indiquées sur les branches. Les trois principaux groupes de séquences $V H$ sont distingués par un code couleur: groupe $A$ en bleu, groupe $B$ en rouge et groupe $C$ en vert. $\dot{A}$ l'extrémité des branches, les cercles indiquent que la SSR associée au segment VH est connue, la couleur du cercle précise le type de SSR concerné. Les séquences autres qu'humaines sont désignées par le nom de genre de l'animal suivi par le numéro d'accès dans Genbank. L'arbre a été enraciné avec les segments VH 101 de requin (Heterodontus francisci) et VH 102 de raie (Raja erinacea), car ces séquences se branchent avant la divergence des segments VH de mammifères [5]. 


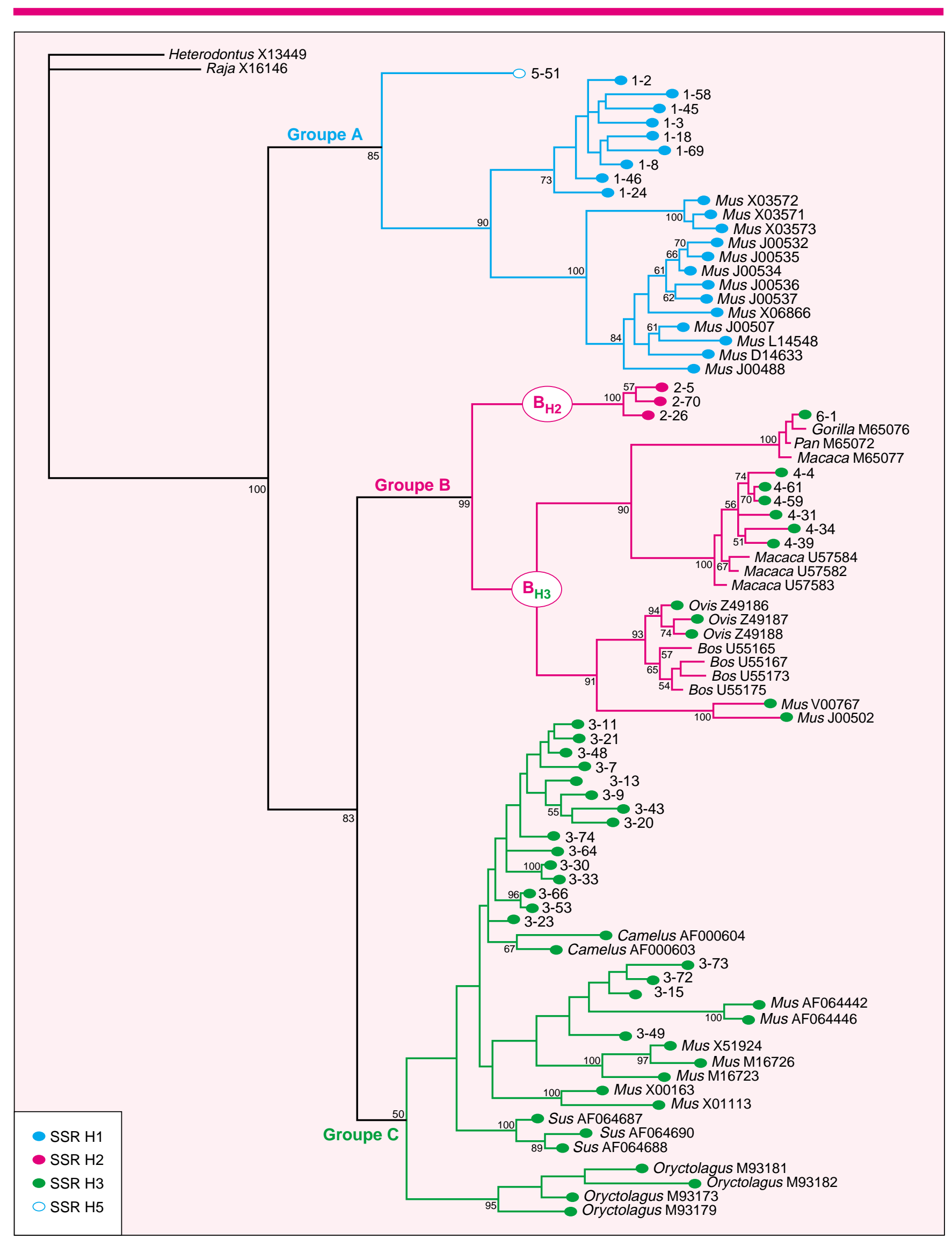

$\mathrm{m} / \mathrm{s} n^{\circ} 11$, vol. 17 , novembre 2001 
codante, sa SSR 3'-adjacente, ainsi que la région 5'-régulatrice de la transcription. Ces trois éléments ont donc probablement évolué comme une unité fonctionnelle, ce qui implique qu'ils partagent la même histoire phylogénétique. Cette hypothèse a été testée en analysant la distribution des différents types de SSR sur l'arbre phylogénétique obtenu avec les séquences VH (figure 3).

Conformément à de précédentes analyses [5-7], trois groupes majeurs de séquences $\mathrm{VH}, \mathrm{A}, \mathrm{B}$ et $\mathrm{C}$, ont été mis en évidence chez les mammifères (figure 3). La présence de séquences VH appartenant à la fois à l'homme et à la souris dans ces trois groupes suggère que leur séparation est antérieure à la radiation des mammifères euthériens, il y a 65 millions d'années (MA) [7]. D'autres analyses ont montré, par ailleurs, que les groupes A et B contiennent des amphibiens en plus des mammifères, alors que le groupe $\mathrm{C}$ inclut aussi des oiseaux, des reptiles, des amphibiens, et des poissons osseux $[5,6]$. Ceci indique que les groupes $\mathrm{A}$, $\mathrm{B}$ et $\mathrm{C}$ avaient déjà divergé avant la radiation des tétrapodes aux alentours de 350-400 MA [5].

Selon le modèle birth-and-death, les trois groupes A, B et C obtenus sur la base des séquences $\mathrm{VH}$ devraient être retrouvés en analysant les SSR. Ce modèle se vérifie dans le cas des groupes A et C (figure 3): les segments $\mathrm{VH}$ du groupe A sont flanqués de SSR de types H1 ou H5, lesquels apparaissent apparentés sur la base des séquences de l'espaceur (mais pas sur la base des régions 3'-adjacentes des SSR, ce qui justifie la définition de deux types différents (figure 2), et tous les segments $\mathrm{VH}$ du groupe C sont liés à des SSR appartenant au seul type H3. En revanche, l'analyse au sein du groupe B ne permet pas d'avaliser le modèle birth-and-death car les segments VH sont associés à des SSR de deux types non apparentés, H2 et H3 (correspondant respectivement aux groupes $\mathrm{B}_{\mathrm{H} 2}$ et $\mathrm{B}_{\mathrm{H} 3}$, figure 3).

\section{Quelle est l'origine des SSR H3 du groupe B?}

Deux hypothèses permettent d'inter-

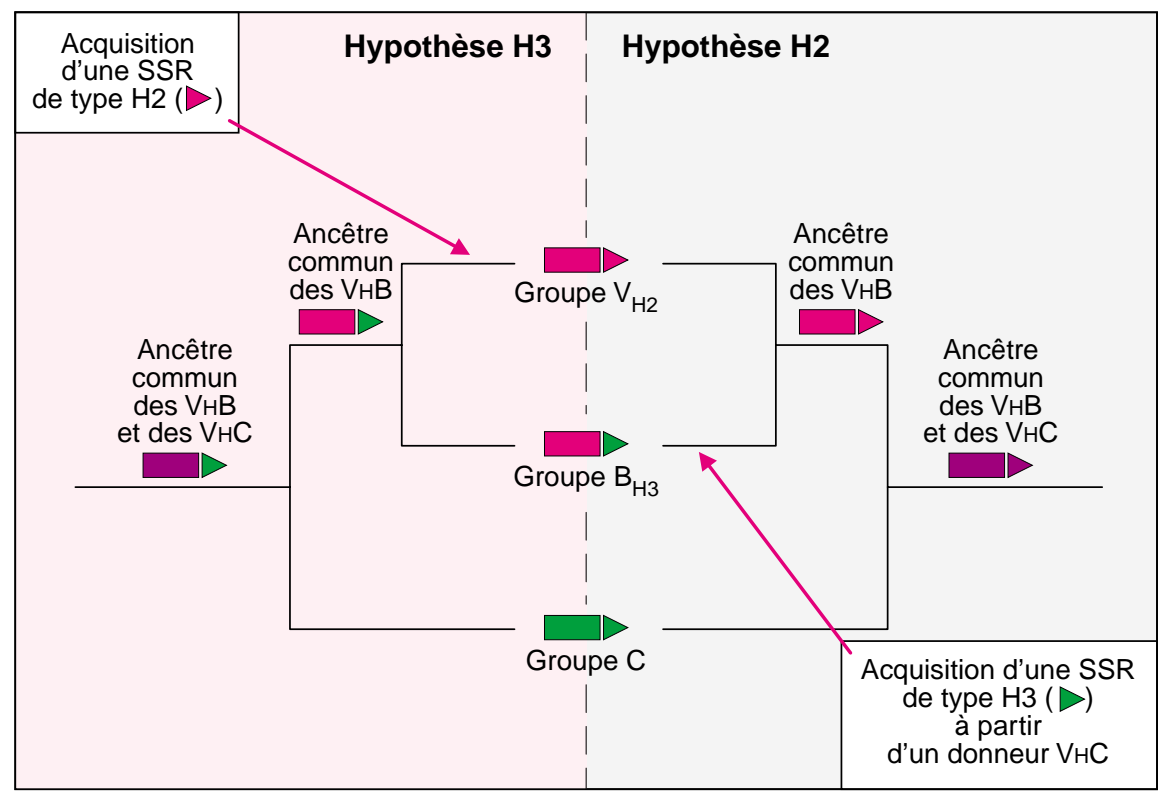

Figure 4. Deux hypothèses pour expliquer I'origine des SSR H3 du groupe B. La première (Hypothèse H3), suppose que le segment $V H$ ancestral du groupe $B$ était connecté à une SSR $\mathrm{H} 3$ et que l'ancêtre du groupe $B_{\mathrm{H}_{2}} \mathrm{~s}^{\prime}$ est doté d'une SSR du type $\mathrm{H} 2$ par un processus qui reste à déterminer. Ceci implique a fortiori que le type de SSR H3 était déjà différencié chez l'ancêtre commun de toutes les séquences $\mathrm{VH}$ des groupes $B$ et $C$. Inversement, la seconde (Hypothèse H2) stipule que le segment $V_{H}$ ancestral du groupe $B$ était lié à une SSR H2 et que l'ancêtre du groupe $B_{H_{3}}$ a obtenu une SSR du type $H 3$ à partir d'un donneur $V H$ du groupe $C$. Les segments de gène $V_{H}$ sont représentés par des rectangles rouges pour ceux du groupe $B$ et verts pour ceux du groupe $C$. Les SSR sont symbolisées par des triangles rouges pour celles du type $\mathrm{H} 2$, et verts pour celles du type H3. La couleur violette est utilisée pour représenter les séquences ancestrales communes aux $\mathrm{VH}$ des groupes $B$ et $C$.

dans le groupe B (figure 4). Selon la première (Hypothèse H3), le segment $\mathrm{VH}$ ancestral du groupe B était connecté à une SSR H3 et l'ancêtre du groupe $\mathrm{B}_{\mathrm{H} 2}$ s'est doté d'une SSR du type H2 par un processus qui reste à déterminer. Inversement, la seconde (Hypothèse H2) stipule que le segment VH ancestral du groupe B était lié à une SSR H2 et que l'ancêtre du groupe $\mathrm{B}_{\mathrm{H} 3}$ a obtenu une SSR du type H3 à partir d'un donneur $\mathrm{VH}$ du groupe $\mathrm{C}$.

Trois arguments soutiennent cette dernière hypothèse et indiquent que l'histoire évolutive du locus VH a été marquée par un échange de SSR entre deux segments $\mathrm{VH}$ des groupes B et C. (1) Puisque les groupes B et C avaient déjà divergé avant la radiation des tétrapodes [5], l'hypothèse H3 suppose une conservation tout à fait exceptionnelle des SSR et des régions 3'-adjacentes. Si tel était le cas, on s'attendrait logiquement à trouver non seulement les SSR de type $\mathrm{H} 3$ dans tous les segments $\mathrm{VH}$ des groupes $\mathrm{B}$ et $\mathrm{C}$ de mammifères, mais aussi dans ceux d'autres groupes de tétrapodes. Pourtant, aucun des segments des groupes B et C décrits à ce jour chez les oiseaux, les crocodiles et les amphibiens ne présente de SSR H3. (2) Sous l'hypothèse H3, il faut supposer que le type $\mathrm{H} 2$ provient d'un donneur d'origine inconnue. Dans le cas de l'hypothèse H2, l'origine du type $\mathrm{H} 2$ est connue: il s'agit du type ancestral du groupe $B$, lequel est le résultat d'un simple processus de divergence nucléotidique à partir de la SSR ancestrale des groupes B et C. (3) La comparaison des groupes $\mathrm{B}_{\mathrm{H} 3}$ et $\mathrm{C}$ révèle que les SSR et les régions 3'-adjacentes sont très proches alors que les régions situées en 5' des SSR ne sont pas apparentées (région 5' régula- 
trice, exon 1, intron et exon 2 des segments $\mathrm{VH})$. De telles différences ne s'expliquent pas par un simple processus de mutation nucléotidique. Seul un événement de recombinaison peut rendre compte de ces différences.

Bien qu'il soit difficile de dater précisément l'événement qui a conduit au remplacement d'une SSR H2 par une SSR H3, trois indices reposant sur des données paléontologiques [8] indiquent qu'il a pu avoir lieu entre 115 et 65 MA. (1) L'absence de SSR H3 chez les amphibiens, reptiles et oiseaux, suggère que l'événement est arrivé après l'émergence des premiers mammifères, aux alentours de 200 MA. (2) Le groupe $\mathrm{B}_{\mathrm{H} 3}$ étant composé d'espèces appartenant à trois ordres différents de mammifères (cétartiodactyles, primates et rongeurs), cela indique que l'événement a pris place avant la radiation adaptative des mammifères euthériens, il y a 65 MA. (3) L'analyse des ADN complémentaires de marsupiaux disponibles dans les banques de données montre qu'aucune séquence VH de Monodelphis domestica et Trichosurus vulpecula n'est liée au groupe B, ce qui laisse penser que l'événement a eu lieu après l'émergence des mammifères euthériens, il $\mathrm{y}$ a environ 115 MA.

\section{Les SSR de type H3 sont-elles plus efficaces pour la recombinaison VDJ ?}

Chez l'Homme, la grande majorité des segments $\mathrm{VH}$ fonctionnels sont associés à des SSR H3 (26 sur un total de 39 séquences, figure 2) alors que les SSR H2 sont très peu représentées (seulement trois exemplaires). Par ailleurs, il est frappant de constater qu'à ce jour toutes les espèces de mammifères pour lesquelles des SSR ont été séquencées possèdent le type H3 (chameau, lapin, mouton, porc et souris), alors que le type H2 n'a été décrit que chez l'homme. De plus, des analyses phylogénétiques incluant les séquences d'ADN complémentaire ont révélé d'autres espèces susceptibles de détenir des SSR H3 (cheval, chien, lama, opossums d'Amérique du Sud et d'Australie, rat et vison). Ces mêmes analyses laissent toutefois penser que des SSR H2 pourraient être découvertes dans le génome du rat et de la souris.

Le succès évolutif des SSR H3 contraste fortement avec la rareté des SSR H2 au sein des mammifères. La comparaison des SSR humaines indique que cette représentation limitée pourrait s'expliquer par l'atypisme de l'heptamère et du nonamère des SSR H2. Alors que l'heptamère CACAGTG caractérise la grande majorité des SSR (toutes celles des types $\mathrm{H} 1$ et $\mathrm{H} 5$, ainsi que 24 des 26SSR du type H3), il est surprenant de constater que l'heptamère du type H2 correspond à CACAAAG ou CACAGAG (figure 2). Des tests fonctionnels ont en effet montré que l'altération de n'importe lequel des nucléotides de l'heptamère CACAGTG entraîne une forte diminution dans les fréquences de recombinaison [9, 10]. Concernant le nonamère, le motif AAA en position 5-7 apparaît primordial pour assurer la fonction de recombinaison $[9,10]$. Ce motif est conservé chez tous les SSR H1, H3 et $\mathrm{H} 5$, et une fois encore le type $\mathrm{H} 2$ apparaît exceptionnel, deux de ses trois représentants ne possédant pas ce motif (figure 2). Les variations de l'heptamère et du nonamère observées dans les SSR H2 suggèrent donc une faible efficacité de ces séquences pour la recombinaison VDJ. En accord avec cette hypothèse, l'analyse des IgM exprimées dans les cellules B humaines circulantes [11] a montré qu'au sein du groupe B, les séquences $\mathrm{VH}$ associées à des SSR H2 étaient sous-exprimées par rapport à leur représentation dans le génome (expression attendue 7,7\%, expression observée $<3 \%$ ), alors que les séquences $\mathrm{VH}$ liées à des SSR H3 étaient davantage exprimées (famille VH4: attendue $15,4 \%$, observée 19$23 \%$ ).

En conclusion, le remplacement d'une SSR H2 par une SSR H3 au cours de l'évolution des mammifères a probablement été sélectionné positivement car les SSR H3 apparaissent plus efficaces pour la recombinaison VDJ. En faveur de cette hypothèse, il faut aussi noter que toutes les espèces caractérisées par un répertoire restreint de segments $\mathrm{VH}$ (cha- meau, porc, lapin et mouton) ne présentent que des SSR de type H3 (figure 3).

\section{Comment s'est produit l'échange de SSR au cours de l'évolution des mammifères ?}

Deux modèles peuvent expliquer la présence de deux types de SSR (H2 et H3) au sein du groupe B (figure 5). Le premier modèle fait intervenir un crossing-over inégal dans les cellules germinales qui aurait entraîné une duplication en tandem sur le chromosome sélectionné au cours de l'évolution (figure $5, I$ ). Ce modèle suppose que la recombinaison homologue puisse se faire entre des séquences $\mathrm{VH}$ divergentes, les groupes B et C étant séparés depuis plus de 350-400 MA [5].

Le second modèle suppose que l'échange de SSR s'est produit par un mécanisme faisant intervenir une reconnaissance des SSR par les protéines RAG (figure 5, II). Ce modèle suppose donc que la recombinaison de type VDJ puisse exceptionnellement avoir lieu dans les cellules germinales des mammifères, comme cela a été montré chez les requins $[12,13]$. Cependant, au lieu d'obtenir une recombinaison standard, c'est-à-dire impliquant deux SSR de tailles différentes (l'une avec un espaceur de $23 \mathrm{pb}$ et l'autre avec un espaceur de $12 \mathrm{pb}$ ) et orientées en sens inverse, le modèle proposé (figure 5, II) prédit que la recombinaison a été réalisée entre deux SSR présentant une taille et une orientation identique. Cette situation particulière ayant déjà été observée expérimentalement [14], rien ne permet d'exclure cette éventualité, d'autant que le site apparent de recombinaison est localisé au niveau de la limite entre la séquence VH et la SSR.

Indépendamment du mécanisme impliqué, il est clair que la possibilité de changement de SSR a été un élément important au cours de l'évolution du locus VH des mammifères. Par ce moyen, des segments de gène présentant de meilleures propriétés de liaison à l'antigène pourraient en effet acquérir un niveau de représentation plus élevé dans le répertoire du système immunitaire 

(A) Modèle I. Crossing-over inégal entre deux segments
VH des groupes B et C

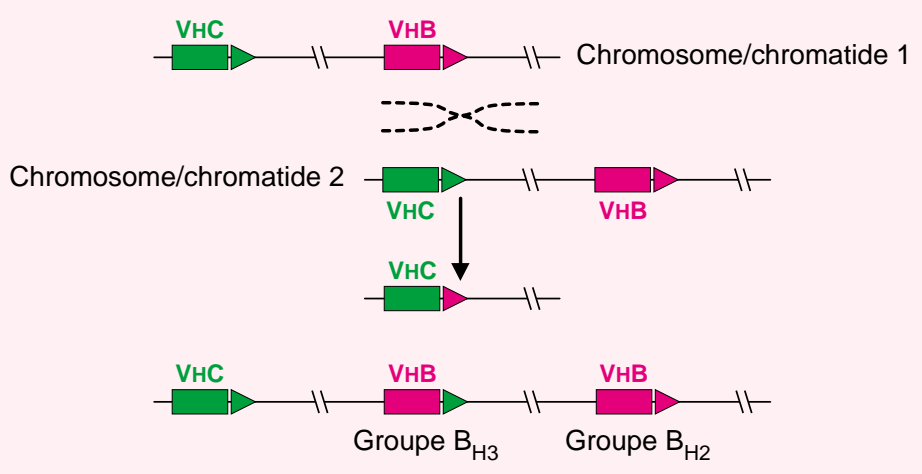

(B) Modèle II. Recombinaison impliquant des séquences "signal de recombinaison"

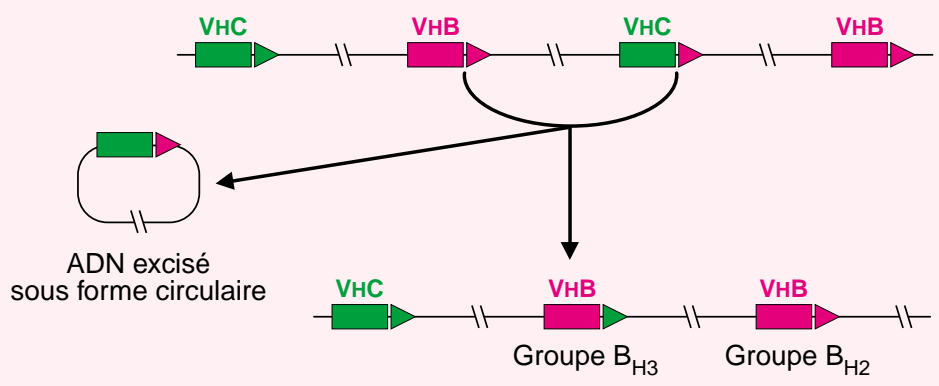

Figure 5. Deux modèles pour expliquer la présence de SSR H3 dans le groupe B. Le premier fait intervenir un crossing-over inégal (modèle l), le second implique un événement de type recombinaison V(D)J (modèle II). Les segments de gène $V H$ sont représentés par des rectangles rouges pour ceux du groupe $B$ et verts pour ceux du groupe C. Les SSR sont symbolisées par des triangles rouges pour celles du type $\mathrm{H2}$, et verts pour celles du type $\mathrm{H} 3$.

\section{Remerciements}

Je remercie le Pr Jean Deutsch et les Drs Rachel Golub et Michaël Manuel pour leurs commentaires sur la première version du manuscrit.

\section{RÉFÉRENCES}

1. Lewis SM. The mechanism of $\mathrm{V}(\mathrm{D}) \mathrm{J}$ joining: lessons from molecular, immunological, and comparative analyses. Adv Immunol 1994; $56: 27-150$.

2. Gellert M. Recent advances in understanding V(D)J recombination. Adv Immunol 1997; 64: 39-64.

3. Matsuda F, Ishii K, Bourvagnet $\mathrm{P}$, et al. The complete nucleotide sequence of the human immunoglobulin heavy chain variable region
4. Hassanin A, Golub R, Lewis SM, Wu GE. Evolution of the recombination signal sequences in the Ig heavy-chain variable region locus of mammals. Proc Natl Acad Sci USA 2000; 97 : 11415-20.

5. Ota T, Nei M. Divergent evolution and evolution by the birth-and-death process in Biol Evol 1994; 11 : 469-82.

6. Nei M, Gu X, Sitnikova T. Evolution by the birth-and-death process in multigene families of the vertebrate immune system. Proc Natl Acad Sci USA 1997; 94: 7799-806.

7. Schroeder HWJ, Hillson JL, Perlmutter RM. Structure and evolution of mammalian VH families. Int Immunol 1990; 2: 41-50.

8. Benton MJ. Vertebrate Palaeontology. Second Édition, Chapman \& Hall, London, 1997: $452 \mathrm{p}$.

9. Hesse JE, Lieber MR, Mizuuchi K, Gellert M. V(D)J recombination: a functional defithe immunoglobulin VH gene family. Mol nition of the joining signals. Genes Dev 1989; 3: 1053-61.

10. Akamatsu Y, Tsurushita N, Nagawa F, et al. Essential residues in V(D) J recombination signals. J Immunol 1994; 153: 4520-9.

11. Rassenti LZ, Kohsaka H, Kipps TJ. Analysis of immunoglobulin $\mathrm{VH}$ gene repertoire by an anchored PCR-ELISA. Ann NY Acad Sci 1995; 764: 463-73.

12. Lee SS, Fitch D, Flajnik MF, Hsu E. Rearrangement of immunoglobulin genes in shark germ cells. J Exp Med 2000; 191 : $1637-48$.

13. Litman GW, Anderson MK, Rast JP. Evolution of antigen binding receptors. Annu Rev Immunol 1999; 17: 109-47.

14. Lewis SM, Hesse JE, Mizuuchi K, Gellert M. Novel strand exchanges in V(D)J recombination. Cell 1988; 55 : 1099-107.

15. Swofford DL. PAUP: Phylogenetic Analysis Using Parsimony, version 3.1.1, Illinois Natural History Survey, Champaign IL, 1993.

16. Hassanin A, Lecointre G, Tillier S. The " evolutionary signal» of homoplasy in protein-coding gene sequences and its consequences for a priori weighting in phylogeny. CR Acad Sci III 1998; 321 : 611-20.

17. Hassanin A, Pasquet E, Vigne JD Systematic relationships within the subfamily Caprinae (Artiodactyla, Bovidae) as determined from cytochrome $b$ sequences. $J$ Mammal Evol 1998; 5 : 217-36.

18. Felsenstein J. Confidence limits on phylogenies: an approach using the bootstrap. Evolution 1985; 39 : 783-91.

\section{* GLOSSAIRE *}

Ig: immunoglobuline.

MA: million d'années.

$p b$ : paire de bases.

$\boldsymbol{R} \boldsymbol{A} \boldsymbol{G}$ : recombination activating gene.

SSR: séquences signal de recombinaison.

TcR: T cell receptor.

\section{Alexandre Hassanin}

Service de biosystématique, Université Pierre-et-Marie-Curie (Paris 6), Bât. B, $7^{e}$ étage, case 241, 9, quai St-Bernard, 75252 Paris Cedex 05, France.

E-mail: Alexandre.Hassanin@snv.jussieu.fr

\section{TIRÉS À PART}

A. Hassanin. 


\section{Summary}

Evolution of the recombination signal sequences

in the immunoglobulin heavy

chain variable region locus

The immunoglobulin (Ig) and $\mathrm{T}$ cell receptor (TCR) loci of vertebrates present an exceptionally dynamic evolutionary history, but the mechanisms responsible remain unknown. Genes encoding the V, D, and $J$ segments of antigen receptors are somatically rearranged in developing lymphocytes by a specialised DNA recombination mechanism called V(D)J recombination. This sitespecific recombination requires the presence of short target sequences, named «Recombination Signal Sequences»(RSS), which are adjacent to the coding segments V, D and J. Recently, V(D)J recombination has been reported in the germline of cartilaginous fishes. Assuming that germline $\mathrm{V}(\mathrm{D}) \mathrm{J}$ rearrangements also occurred during the mammal history, we expect to find that coding segments and RSSs were not always associated as a functional unit during evolution. In other words, the phylogenetic tree constructed from coding segments could be different from the one produced with the RSSs. This possibility is here examined by studying the complete human Ig heavy chain variable region $(\mathrm{VH})$ locus. In this locus, four distinct types of RSS have been defined, namely $\mathrm{H} 1, \mathrm{H} 2, \mathrm{H} 3$, and H5. No other RSS type has been detected in other mammalian species. The phylogenetic analyses, performed on one hand on the coding VH segments and on the other hand on the adjacent RSSs, revealed that the type H2 RSS of one VH gene has clearly become replaced by a type H3 RSS during mammalian evolution. Two general models are proposed to explain the RSS swap: the first involves an unequal crossingover, the second implicates germline activation of $\mathrm{V}(\mathrm{D}) \mathrm{J}$ recombination. The recombination event has likely been selected during the evolution of mammals because the type H3 RSS seem to provide better VDJ recombination efficiency than the type H2 RSS.

\section{$1^{\text {res }}$ Assises de Génétique Humaine et Médicale \\ 18-20 janvier 2002 \\ Palais du Pharo \\ Marseille, France}

\section{Séances plénières}

Génétique des maladies neuromusculaires

Gisèle Bonne, Ketty Schwartz, Judith Melki, Nicolas Lévy

Instabilité chromosomique et cancer

Alain Aurias, Annick Harel-Bellan

Génétique et infectiologie

Dominique Dormont,

Éric Denamur, Laurent Abel

Génétique du développement

Pierre Chambon, Patrick Charnay, Olivier Pourquié, Michel Vekemans

\section{Sessions simultanées}

\section{Génétique clinique}

Du phénotype au génotype : le rôle du clinicien

Génétique chromosomique

Les nouveaux enjeux

de la cytogénétique moléculaire

Génétique moléculaire

Physiopathologie

des maladies génétiques:

aspects moléculaires

Fotopathologie

Valeur prédictive

des examens fretopathologiques : implications

dans le conseil génétique

\section{Conférence d'actualité}

Thérapie génique

Alain Fischer

\section{Secrétariat scientifique}

Hôpital d'Enfants de la Timone

Département de génétique médicale

Nicole Philip, Nicolas Lévy

13385 Marseille Cedex 5, France

Tél. : 0491386734

Fax : 0491386973

e-mail : secretariat.scientifique

@ assises-genetique.org 